\title{
13 Männer als Palliativpatienten
}

\author{
Heide Götze, Norbert Köhler, Lutz Gansera, Stephanie Berger \\ und Susanne Singer
}

„Der Tod hat [...] in der Lebenswelt des Alltags eine besondere Stellung und Bedeutung: Er ist die problematischste und am wenigsten eindeutig verstehbare Grundtatsache des Daseins." (Nassehi u. Weber 1989, S. 180)

Will man die Situation von Männern in palliativen Behandlungssituationen besser verstehen, muss man sich mit der besonderen gesellschaftlichen Rolle von Sterbenden beschäftigen. War das Sterben früher noch eine eher öffentliche Angelegenheit, wurde der Tod im Laufe der Menschheitsgeschichte als Vorgang und als Gedanke zunehmend hinter die Kulissen des Gesellschaftslebens verlegt (vgl. auch Elias 1991). Heutzutage werden die Menschen in den Medien täglich mit vielen Toten und unzähligen Todesarten konfrontiert, haben jedoch persönlich nur wenig Berührung mit Sterbenden oder einem Leichnam gehabt (Student et al. 2004). Dabei betrifft der Tod jeden von uns und wird doch als etwas erlebt, was nur anderen geschieht. Auch ist eine zunehmende Institutionalisierung des Sterbens zu beobachten: Zwei Drittel der Menschen sterben heute in Institutionen wie Krankenhäusern oder Pflegeheimen. Durch die wachsenden Möglichkeiten der modernen Medizin, Leben zu verlängern, erscheint der Tod häufig als Niederlage und nicht als natürlicher Vorgang, der Teil des menschlichen Lebens ist. Die Hospizbewegung hat sich zum Ziel gesetzt, das Sterben als Bestandteil des Lebens in unsere Gesellschaft zurückzuholen und es wieder zu enttabuisieren (Nagele u. Feichtner 2009).

\subsection{Die palliative Situation}

\section{Definition}

Ein Palliativpatient ist ein Mensch mit einer nicht heilbaren progredienten und weit fortgeschrittenen Erkrankung mit begrenzter Lebenserwartung, für den das Hauptziel der Behandlung die Lebensqualität ist.

Wenn ein Mann an einer unheilbaren Erkrankung leidet, erlebt er in der Regel eine existenzielle Bedrohung von hoher emotionaler Intensität. Der Zeitpunkt, ab dem 
eine Behandlung mit dem Etikett „palliativ“ versehen wird, bestimmt sich von den körperlichen Gegebenheiten her. Im Erleben der Patienten findet sich dazu kein unmittelbares Korrelat, auch wenn sich das Befinden aufgrund der körperlichen Situation langfristig verschlechtern wird und sich Gedanken an das Sterben und den Tod aufdrängen. Den Zustand der Unheilbarkeit erlebt der Patient weniger aus sich selbst heraus, als dass er von außen davon erfährt. Es kann allerdings nicht davon ausgegangen werden, dass ein verstandesmäßiges Anerkennen der palliativen Lage auch unmittelbar emotional nachvollzogen wird und eine Einwilligung in den Sterbeprozess bedeutet.

Das Wissen, dass man eine unheilbare Krankheit hat, ist für den Patienten und auch für dessen Angehörige meist eine schwer erträgliche Vorstellung. Die Reaktionen darauf sind individuell verschieden und spiegeln sich im seelischen Befinden, im sozialen Kontakt und im körperlichen Beschwerdenbild (Schwarz u. Singer 2008).

Wie kann ich einen Mann in der Palliativsituation unterstützen? (Schwarz $u$. Singer 2008, S. 78)

- Schaffung einer verlässlichen Struktur durch überschaubare Routinen in Behandlung und Pflege sowie personelle Konstanz

- Vorbeugen von sozialer Isolation durch Erleichterung und Verbesserung der sozialen Kontakte

- Reduktion von Hilflosigkeit und Kontrollverlust durch die Förderung von Autonomie bei Berücksichtigung der jeweiligen Rollenerwartungen seitens des Mannes

- Förderung des Ausdrucks von Bedürfnissen und Emotionen sowie des Bedürfnisses nach Sinnfindung und Spiritualität

\subsection{Der sterbende Mann}

Das Gefühl, eine Last zu sein und seine Unabhängigkeit zu verlieren ist für alle Menschen in einer palliativen Situation ein relevantes Thema. Bei männlichen Palliativpatienten scheint dies jedoch eine besondere Bedeutung zu erlangen (Broom u. Cavenagh 2010). Ein Mensch zu sein, der auf die Hilfe von anderen angewiesen ist, ist für die betroffenen Männer oft mit Trauer über den Verlust ihres Status' verbunden. Männer haben eher Schwierigkeiten, Hilfe von Anderen anzunehmen. Die palliative Situation ist nur schlecht mit den üblichen Vorstellungen vom Mannsein und der Idealvorstellung von einem schnellen und selbstbestimmten Tod vereinbar (Kelly et al. 2002). So existiert noch in vielen Köpfen die Vorstellung, dass ein Mann an einem Herzinfarkt sterben sollte.

Linnemann (2005) beschreibt in seinen Beobachtungen, dass Frauen mit mehr Bewusstsein und Offenheit und einem klareren Blick in die letzte Lebensphase gehen als Männer. Frauen seien eher dazu bereit, noch nicht Gesagtes zu thematisieren, wo hingegen Männern oft die Worte fehlten. Auch wirken Männer häufig überfordert mit der Tatsache ihres nahenden Lebensendes. Während Frauen eine deutlichere Hingabe an den Sterbeprozess und den Tod zeigten, scheinen Männer dazu zu neigen, auch in dieser Phase des Lebens noch gegen etwas ankämpfen zu müssen. Auch 
im Hospiz seien Männer wesentlich häufiger als Frauen bis kurz vor ihrem Tod in umtriebiger Bewegung. Laut Hollstein (2004) sind viele sterbende Männer weiterhin auf „Tun und Haben“ fixiert und nicht auf „Lassen und Sein“.

Seelische Bedürfnisse in der letzten Lebensphase (Schwarz u. Singer 2008, S. 80)

- Bewältigung der Verletzung des Selbstwertgefühls infolge von körperlichen Veränderungen, von Abhängigkeit und Hilfsbedürftigkeit

- Überwindung und Auflösung negativer Gefühle gegenüber der Vergangenheit und der Gegenwart

- Verwirklichung des Glaubens, ein sinnvolles Leben gelebt zu haben, eine positive Lebensbilanz ziehen zu können und bei den Hinterbliebenen in guter Erinnerung zu bleiben

- Trauer über den Verlust der äußeren Welt und Wiederbelebung guter und tragender Erinnerungen und Vorstellungen, die eine Einordnung des Selbst in ein Sinn-Ganzes erlauben

- Erledigung begonnener Aufgaben, denen sich der Kranke verpflichtet fühlt, verbunden mit der Sorge um und für die „Hinterbleibenden“

In der letzten Zeit vor dem Tod sind eine ständige pflegerische Präsenz und eine hohe ärztliche Kompetenz erforderlich, um die zahlreichen körperlichen Symptomzustände wie starke Schmerzen, Ernährungs- und Verdauungsprobleme, Schlafstörungen und auch Verwirrtheitszustände wirksam kontrollieren zu können. Vor allem sterbende Patienten setzten sich nahezu regelmäßig - vielfach auch unbewusst - mit einer Reihe von Problemen auseinander. Die psychischen Ausdrucksformen werden radikaler, unvermittelter und instabiler.

Sterbende Menschen richten ihr Interesse zunehmend von außen nach innen. Auch wenn der Eindruck von Passivität entsteht, ist dies vermutlich ein höchst aktives Geschehen, bei dem der Sterbende ganz mit sich selbst beschäftigt ist (Nageleu. Feichtner 2009).

Am Lebensende sind Sicherheit gebende Umweltbedingungen, verlässliche und wertschätzende mitmenschliche Beziehungen sowie eine erträgliche Symptombelastung von entscheidender Bedeutung.

\subsection{Komorbidität bei Palliativpatienten}

Bisherige Studien zur Symptombelastung bei Krebspatienten berichten, dass männliche Patienten weniger stark über körperliche und psychische Beschwerden klagen als weibliche Patienten (Schwarz u. Hinz 2001; Herschbach 2006). Die Prävalenz von Schmerz, Depression und Delirium nimmt jedoch unabhängig vom Geschlecht mit fortschreitender Krankheit zu. Dazu können weitere Symptome kommen wie starke Luftnot, Ödeme, Verstopfung und Übelkeit. Etwa drei Viertel der Palliativpatienten leiden zudem unter Schwäche und Müdigkeit (Perner et al. 2010). Risikofaktoren für Anpassungsstörungen und Depressionen sind eine schlechte körperliche Verfassung, 
die Sorge, anderen zur Last fallen zu können sowie Unzufriedenheit mit der sozialen Unterstützung (Akechi 2004). Krebspatienten mit Schmerzen entwickeln doppelt so häufig psychische Symptome (39\%) als Patienten ohne Schmerzen (19\%) (Schwarz u. Singer 2008). Auch die zunehmende Immobilität und die damit einhergehenden Einschränkungen werden von den Patienten als hinderlich beschrieben. Diese Umstände haben zur Folge, dass die pflegerische und medizinische Behandlung von Palliativpatienten oft von einer notwendigen und mitunter auch sehr belastenden Gefühlsund Interaktionsarbeit überlagert wird.

In der palliativen Behandlung steht die Lebensqualität des Patienten im Mittelpunkt. Neben der Kontrolle physischer, somatopsychischer und psychischer Symptome sind die Berücksichtigung der individuellen Bedürfnisse und spirituellen Anliegen sowie die Einbeziehung der Angehörigen des Patienten von großer Bedeutung.

\subsection{Palliative Versorgung in Deutschland}

\subsubsection{Versorgungssituation}

In den vergangenen 30 Jahren ist in Deutschland ein umfassendes Netz von Einrichtungen der Hospiz- und Palliativversorgung entstanden. So gibt es in Deutschland aktuell etwa 1.500 ambulante Hospiz- und Palliativdienste, 231 Palliativstationen und 179 stationäre Hospize (Deutscher Hospiz- und Palliativverband 2011). Nur etwa ein Viertel der Menschen, die in einem Pflegeheim und ein Drittel der Menschen, die in einem stationären Hospiz versterben, sind männlich (Cruel et al. 2009; Linnemann 2005). Linnemann (2005) merkt dazu an, dass Männern oft die Grundvoraussetzung zur Aufnahme ins Hospiz fehle: die Einsicht in die Unheilbarkeit ihrer Erkrankung und das innere Einverständnis, im Hospiz zwar Linderung der Symptomatik erfahren zu können, aber nicht mehr Heilung. Der hohe Frauenanteil im Hospiz hängt aber auch mit der höheren Lebenserwartung der Frauen zusammen, die zur Folge hat, dass Frauen in ihren letzten Lebensjahren häufig allein leben und eine häusliche Versorgung schwieriger zu realisieren ist.

\subsubsection{Häusliche Palliativversorgung}

Männliche Palliativpatienten werden häufiger zu Hause versorgt als schwerkranke Frauen, was neben demografischen Gegebenheiten auch mit der höheren Bereitschaft von Frauen zusammenhängt, die Versorgung eines pflegebedürftigen Angehörigen zu übernehmen (Gräßel 1998). Die meisten Menschen in einer palliativen Situation haben den Wunsch, die verbleibende Lebenszeit beschwerdefrei und würdevoll in ihrer vertrauten Umgebung zu verleben. Inwieweit das zu realisieren ist, hängt von verschiedenen Faktoren ab. Kinder wohnen und arbeiten heute oft weiter entfernt von den Eltern als früher, haben flexiblere und längere Arbeitszeiten, und so sehen sich viele Angehörige außerstande, die Verantwortung für eine oft mehrmonatige, häusliche Rund-um-die-Uhr-Pflege zu übernehmen. Kann die Versorgung des Palliativpatienten in der häuslichen Umgebung personell, räumlich und finan- 
ziell ermöglicht werden, so verteilt sich die Betreuung und Pflege in der Regel auf nur wenige Schultern. Dabei geraten die pflegenden Angehörigen oft körperlich und psychisch an ihre Grenzen oder fühlen sich bei Symptomverschlechterung häufig überfordert. Gründe für ein Scheitern häuslicher Palliativversorgung sind unter anderem die noch nicht flächendeckende Umsetzung der Richtlinien zur „Spezialisierten ambulanten Palliativversorgung“ ( $\$ 37 \mathrm{~b}$, Abs. 1, SGB V), mangelnde Kooperation zwischen den Behandlern sowie unzureichende Schmerztherapie und Symptomkontrolle (Götze et al. 2010; Deutscher Bundestag 2005; Schneider et al. 2005).

\section{Bei der Diskussion über den Mann als Palliativpatient darf nicht vergessen werden, dass die geschlechtliche Identität ein Element ist innerhalb der kom- plexen Reihe von Themen, einschließlich der Persönlichkeit, der Kultur und den zwischenmenschlichen Beziehungen. Trotz der geschlechtsspezifischen Besonderheiten ist Sterben immer individuell und nicht normierbar.}

\section{Literatur}

Akechi T, Okuyama T, Sugawara Y, Nakano T, Shima Y, Uchitomi Y (2004) Major depression, adjustment disorders, and post-traumatic stress disorder in terminally ill cancer patients: associated and predictive factors. I Clin Oncol 22, 1957-1965

Broom A, Cavenagh I (2010) Masculinity, moralities and being cared for: an exploration of experiences of living and dying in a hospice. Soc Sci Med 71, 869-876

Cruel E, Kroll B, Bardehle D (2009) Zukunftsperspektiven ambulanter Palliativversorgung in Bielefeld. Gesundheitswesen 71, A56

Deutscher Bundestag (2005) Zwischenbericht der Enquete-Kommission Ethik und Recht der modernen Medizin Verbesserung der Versorgung Schwerstkranker und Sterbender in Deutschland durch Palliativmedizin und Hospizarbeit. Drucksache 15/5858 2005

Deutscher Hospiz- und Palliativverband (2011) Hospiz- und Palliativversorgung. http://www.dhpv.de/themen_ hospiz-palliativ.html; abgerufen am: 25.07.2011

Elias N (1991) Über die Einsamkeit der Sterbenden in unseren Tagen, Suhrkamp Verlag, Frankfurt am Main

Ensink FBM, Bautz MT, Hanekop GG (2001) Optimierung der ambulanten palliativmedizinischen Betreuung terminal kranker Tumorpatienten am Beispiel SUPPORT - ethisch zu präferierende Alternative zur Forderung nach aktiver Sterbehilfe. Anästhesiol Intensivmed Notfallmed Schmerzther 36, 530-537

Götze H, Perner A, Anders D, Brähler E (2010) „Die Kommunikation untereinander ist häufig nicht vorhanden“Interviews mit Pflegedienstmitarbeitern zur ambulanten Palliativversorgung. Gesundheitswesen 72 , e60-e64

Gräßel E (1998) Häusliche Pflege demenziell und nicht demenziell Erkrankter. Teil I: Inanspruchnahme professioneller Pflegehilfe. Z Gerontol Geriat 31, 52-56

Herschbach, P (2006) Behandlungsbedarf in der Psychoonkologie. Grundlagen und Erfassungsmethoden. Der Onkologe 12, 41-47

Hollstein W (2004) Geschlechterdemokratie. Männer und Frauen: Besser miteinander leben. VS Verlag für Sozialwissenschaften, Wiesbaden

Kelly B, Burnett P, Pelusi D, Badger S, Varghese F, Robertson M (2002) Terminally ill cancer patients' wish to hasten death. Palliative Medicine 16, 339-345

Linnemann G (2005) Sterben Männer anders? Sozial Extra 29, 16-18

Nagele S, Feichtner A (2009) Lehrbuch der Palliativpflege. Facultas Verlag, Wien

Nassehi A, Weber G (1989) Tod, Modernität und Gesellschaft. Entwurf einer Theorie der Todesverdrängung, Opladen, Westdeutscher Verlag 
Perner A, Götze H, Stuhr C, Brähler E (2010) Ambulante Palliativversorgung von Tumorpatienten im Raum Leipzig. Schmerz 24, 38-45

Robert-Koch-Institut (2010) Krebs in Deutschland 2005/2006. Häufigkeiten und Trends. Robert-Koch-Institut, Berlin

Schneider N, Buser K, Janus K, Brandes I, Amelung VE (2005) Konzepte zur bedarfsgerechten Strukturierung der Palliativversorgung im deutschen Gesundheitswesen: Das Beispiel des Bundeslandes Niedersachsen. Gesundheitswesen $67,755-762$

Schwarz R, Hinz A (2001) Reference data for the quality of life questionnaire EORTC QLQ-C30 in the general German population. Eur | Cancer 37, 1345-1351

Schwarz R, Singer S (2008) Einführung Psychosoziale Onkologie. Ernst Reinhardt Verlag, München

Student JC (2004) Soziale Arbeit in Hospiz und Palliative Care. Reinhardt Verlag, München 